Pacific Journal of Mathematics

A THEOREM ON SIMULTANEOUS OBSERVABILITY 


\section{A THEOREM ON SIMULTANEOUS OBSERVABILITY}

\section{TOM LOUTON}

It is well known in abstract quantum mechanics, that when two observables have a joint distribution, a state can be found, in which both observables have a small variance. In order to consider a converse of this, a relation between two observables is defined. Say $x$ and $y$ are two observables and that when $\alpha$ is a state such that the variance of $x$ in $\alpha$ is small implies the variance of $y$ in $\alpha$ will be small. From the above relation it follows that there is a continuous function $f$, such that $f$ maps the spectrum of $x$ onto the spectrum of $y$. Further, $y=f(x)$.

To make this notion clear, let us consider a commonly used technique to observe natural phenomena accurately, namely, a statistical ensemble, to which a sorting mechanism has been applied.

Say, for example, that observable $A$ is of interest. Let $a$ be the property that a measurement of $A$ is in $E$, a Borel subset of $R$. Let $f=\chi_{E}$, then the probability of $a$, in any state, is equal to the probability of a yes answer to the question $f(A)$. Hence, if we have a subensemble which possesses property $a$, and if $\alpha$ is its state, then $\operatorname{Var}_{\alpha} f(A)=0$.

Suppose one wishes to find accurate values for the observable $B$ at the same time (in the same state) we have knowledge of $A$. One resolves the ensemble re $A$, then the subensemble which has property $a$ is resolved re $B$. However, in general, such a programm will not work. This paper attempts to show that in the event such a programm can be successfully carried through, it can be concluded that $A$ and $B$ actually have a joint distribution.

Before beginning in earnest, we must establish some notation and state an important formula.

The logic, $\mathscr{E}$, and the state set, $\mathfrak{S}$, with which we will work have the following properties:

(1) They satifify Axioms I through VI and VIII of Mackey (1963)-that is, all, but the Hilbert Space Axiom. We will feel free to use the structure and vocabulary developed by Mackey and also to be found in Varadarajan (1962).

(2) Let $a \in \mathscr{E}$ and let us define a set, $S_{a}$ as follows:

$$
S_{a}=\{\alpha \mid \alpha(\alpha)=1\}
$$

If $a \neq 0$, the above axioms assure us that $S_{a} \neq 0$. This axiom assures us that if $a, b \in \mathscr{E}$ and $S_{a} \leqq S_{b}$ then $a \leqq b$. 
We write for the mean of an observable, $x$, in the state $\alpha$,

$$
\mu_{\alpha}(x)=\int t d \alpha x(t)
$$

and we write

$$
\operatorname{Var}_{\alpha}(x)=\int\left(t-\mu_{\alpha} x\right)^{2} d_{\alpha} x
$$

for the variance of $x$ in the state $\alpha$.

We recall that the Axiom III of Mackey deals with mixtures of states. If $\alpha$ is a mixture, we write $\alpha=\Sigma t_{1} \alpha_{1}$, where $\Sigma t_{i}=1$, $t_{i}>0$. We then have the following Variance Formula:

Let $\alpha=\sum_{i=1}^{n} t_{i} \alpha_{i}$ with $\sum_{i=1}^{n} t_{i}=1$ and $t_{i}>0$. Then

$$
\operatorname{Var}_{\alpha}(x)=\Sigma t_{i} \operatorname{Var}_{\alpha_{i}}(x)+\sum_{i<j} t_{i} t_{j}\left(\mu_{\alpha_{i}}(x)-\mu_{\alpha_{j}}(x)\right)^{2} .
$$

The proof of this is straightforeward.

"Definition of $x \rightarrow y$ and a Proof of the Implied Existence of $f$ "

Now, we want to return to the problem of simultaneously accurate measurements. A system is in a state $\alpha$, in which the measurement of $x$ is accurate, if the variance of $x$ is small. Thus, we expect with a probability of near 1 , that a measurement of $x$ will be near $\mu_{\alpha}(x)$. Then, in the context of a statistical ensemble $S_{n}$, we let $\alpha_{i}$ be the state of the subensemble, ${ }_{i} S_{n}$, where we have $\operatorname{Var}_{\alpha_{i}}(x)<\delta$ and $\left|\mu_{\alpha_{i}} x-t\right|<\delta$ where $t \in \sigma(x)$, the spectrum of $x$. Assume that the small variance and the proximity of $\mu_{\alpha_{i}}(x)$ to $t$ implies that $\operatorname{Var}_{\alpha_{i}}(y)<\varepsilon$. We then would have that a resolution of the ensemble, $S_{n}$, with respect to $x$ is also a resolution with respect to $y$. We will, with this view of the problem, proceed to the following

Definition. Assume that $x$ and $y$ are observables. We will write $x \rightarrow y$ on $\sigma(x) / N$ provided that the following conditions hold: There is an $x$-null set, $N$, (possibly empty) such that for each $\varepsilon>0$ and each $\lambda \in \sigma(x) / N$, there exists $\delta_{\lambda}>0$, such that whenever $\alpha$ is a state for which

$$
\left|\mu_{\alpha}(x)-\lambda\right|<\delta_{\lambda}
$$

and

$$
\operatorname{Var}_{\alpha}(x)<\delta_{\lambda},
$$

then

$$
\operatorname{Var}_{\alpha}(y)<\varepsilon .
$$


This definition has the interesting consequence that any two observables so related, implies that there is a function $f: \sigma(x) \rightarrow \sigma(y)$. We will prove this as well as some of the function's interesting properties.

Proposition 1. Suppose $x \rightarrow y$ and that $\left\{\alpha_{n}\right\}$ is a sequence of states such that

$$
\lim _{n \rightarrow \infty} \mu_{\alpha_{n}}(x)=\lambda
$$

for some $\lambda \in \sigma(x)$. If

$$
\lim _{n=\infty} \operatorname{Var}_{\alpha_{n}}(x)=0
$$

then $\mu_{\alpha_{n}}(y)$ is a Cauchy sequence.

Proof. Use the variance formula on a mixture of states $\alpha_{n}$ and $\alpha_{m}$. Show that if $N$ is sufficiently large, $n, m \geqq N$ implies the variance of $x$ in the mixture is small. Thus, by $x \rightarrow y$, the variance of $y$ in the mixture is small. Thus, (recall that the terms of the Variance Formula are all positive) $\left|\mu_{\alpha_{n}}(y)-\mu_{\alpha_{m}}(y)\right| \leqq \varepsilon$.

Proposition 2. Let $x$ and $y$ be observables with $x \rightarrow y$ and let $N$ be an $x$-null set as in the definition of $x \rightarrow y$. Let $\lambda \in(x) / N$ and assume that $\left\{\alpha_{n}\right\}$ and $\left\{\beta_{n}\right\}$ are sequences of states such that

$$
\lim \mu_{\alpha_{n}}(x)=\lim \mu_{\beta_{n}}(x)=\lambda
$$

and

$$
\lim \operatorname{Var}_{\alpha_{n}}(x)=\lim \operatorname{Var}_{\beta_{n}}(x)=0,
$$

then

$$
\lim \mu_{\alpha_{n}}(y)=\lim \mu_{\beta_{n}}(y) .
$$

Proof. Consider a mixture of $\alpha_{n}$ and $\beta_{m}$. Use the Variance Formula on it and show that the variance of $x$ in the mixture is small when $n$ and $m$ are greater than $N$, and $N$ is sufficiently large. Also show that the mean of $x$ in the mixture is near $\lambda$. Thus, by $x \rightarrow y$, the varinace of $y$ is small, from which it follows that $\left|\mu_{\alpha_{n}}(y)-\mu_{\beta_{m}}(y)\right| \leqq \varepsilon$.

Proposition 3. Assume that $x \rightarrow y$ and let $\left\{\alpha_{n}\right\}$ be a sequence of states as in Proposition 2. Let $\gamma=\lim \mu_{\alpha_{n}}(y)$, then $\lambda \in \sigma(y)$.

Proof. For any $\varepsilon>0$, consider Chebychev's inequality. 


$$
\alpha(y)\left(\left\{z|| z-\mu_{\alpha}(y) \mid \geqq \varepsilon\right\}\right) \leqq \operatorname{Var}_{\alpha}(y) / \varepsilon^{2}
$$

Thus,

$$
\alpha(y)\left(\mu_{\alpha}(y)-\varepsilon / 2, \mu_{\alpha}(y)+\varepsilon / 2\right) \geqq 1-4 \operatorname{Var}_{\alpha}(y) / \varepsilon^{2} .
$$

Further, if

a) $\left|\mu_{\alpha}(y)-\gamma\right|<\varepsilon / 2$, then

$$
\left(\mu_{\alpha}(y)-\varepsilon / 2, \mu_{\alpha}(y)+\varepsilon / 2\right) \subset(\gamma-\varepsilon, \gamma+\varepsilon)
$$

Thus, we find $\alpha$ such that (a) holds and such that $4 \operatorname{Var}_{\alpha}(y)<1$, then, $\alpha(y)\left(\mu_{\alpha}(y)-\varepsilon, \mu_{\alpha}(y)+\varepsilon\right) \neq 0$. Hence, for any $\varepsilon>0$, we can find a state $\alpha$, such that $\alpha(y)(\gamma-\varepsilon, \gamma+\varepsilon) \neq 0$, which implies that $\gamma \in \sigma(y)$.

Proposition 4. Say $x \rightarrow y$, on $\sigma(x) / N$. Then there exists a function, $f$, such that

$$
f: \sigma(x) \longrightarrow \sigma(y) .
$$

Proof. Say $\lambda \in \sigma(x) / N$. Then there exists $\left\{\alpha_{n}\right\}$ such that $\lim \mu_{\alpha_{n}}(x)=$ $\lambda$ and $\lim \operatorname{Var}_{\alpha_{n}}(x)=0$. We define $f(\lambda)=\lim \mu_{\alpha_{n}}(y) . \quad f$ is well defined through Proposition 2. By Proposition 3, $f(\sigma(x)) \subset \sigma(y)$.

Proposition 5. $f$ is continuous in the topology induced by the usual topology on $R$.

Proof. Say $\lambda \in \sigma(x) / N$ and $\varepsilon>0$. Then chose $\delta_{\lambda}>0$ corresponding to $\varepsilon^{2} / 36$ in the definition of $x \rightarrow y$. If $\lambda_{1} \in \sigma(x)$ such that $\left|\lambda_{1}-\lambda\right|<\delta_{\lambda} / 3$, find $\alpha$ and $\beta$ states such that $\left|\mu_{\alpha}(x)-\lambda\right|<\delta_{\lambda} / 3, \operatorname{Var}_{\alpha} x<\delta_{\lambda} / 2$, and such that $\left|\mu_{\alpha}(y)-f(\lambda)\right|<\varepsilon / 3$ and that the same relations hold with respect to $\beta$ and $\lambda_{1}$. Thus the variance of $x$ in any mixture of $\alpha$ and $\beta$ is less then $\delta_{\lambda}$ and further the average is with in $\delta_{\lambda}$ of $\lambda$. Hence the variance of $y$ in any mixture of the two is less than $\varepsilon^{2} / 36$. Take a mixture of $1 / 2$ of each and use the variance formula to show $\left|\mu_{\alpha}(y)-\mu_{\beta}(y)\right|<\varepsilon / 3$. Then $\left|f(\lambda)-f\left(\lambda_{1}\right)\right|<\varepsilon$ follows.

Thus we have that the relation of Definition 1 implies the existence of a continuous function $f: \sigma(x) / N \rightarrow \sigma(y)$. We will now consider this relation in the case where $x$ has a discrete spectrum.

$$
\text { “ } x \longrightarrow y \text { Implies } y=f(x) "
$$

Assume that $x$ is an arbitrary observable with a countable spectrum. Let $N$ be the set of all $\lambda$ in the spectrum of $x$ such that $x(\lambda)=0 . \quad N$ is thus $x$-null. Further, any other $x$-null set will 
be in $N$. We assume now that $y$ is an observable such that $x \rightarrow y$ on $\sigma(x) / N$. We will show then that in this case, $y=f(x)$.

Lemma 1. If $x$ and $y$ are as above, $\lambda \in \sigma(x)$ implies

$$
x(\lambda) \leqq y(f(\lambda))
$$

Proof. We have that $\lambda \in \sigma(x) / N$ implies that

$$
x(\lambda) \neq 0 \text { in } \mathscr{E} \text {. }
$$

Thus there is an $\alpha$ such that

$$
\alpha(x(\lambda))=1
$$

Therefore, $\operatorname{Var}_{\alpha}(x)=0$ and that $x \rightarrow y$, we have that $\operatorname{Var}_{\alpha}(y)=0$ and thus by Proposition 4 we have that $\mu_{\alpha}(y)=f(\lambda)$. Now if $\alpha$ is any state such that $\operatorname{Var}_{\alpha}(x)=0$ then $\operatorname{Var}_{\alpha}(y)=0$ and so

$$
\alpha(x(\lambda))=1
$$

implies

$$
\alpha(y(f(\lambda)))=1
$$

Therefore,

$$
S_{x(\lambda)} \subset S_{y(f(\lambda))}
$$

Hence,

$$
x(\lambda) \leqq y(f(\lambda))
$$

TheOREM 1. Let $x \rightarrow y$ as in Lemma 1 , then

$$
y=f(x) \text {. }
$$

Proof. By Lemma 1, and the fact that $x$ is a $\sigma$-homomorphism we have

$$
x\left(f^{-1}(\gamma)\right)=U\left\{x(\lambda) \mid \lambda \in f^{-1}(\gamma)\right\} \leqq y(\gamma)
$$

for all $\gamma \in \sigma(y)$.

Now both $x$ and $y$ are $\sigma$-homomorphisms, so we have

$$
1=U\left\{x\left(f^{-1}(\gamma)\right) \mid \gamma \in \sigma(y)\right\} \leqq U\{y(\gamma) \mid \gamma \in \sigma(y)\} .
$$

Further,

$$
\cup\left\{x\left(f^{-1}(\gamma)\right) \mid \gamma \in \sigma(y) / \gamma^{\prime}\right\} \leqq U\left\{y(\gamma) \mid \gamma \in \sigma(y) / \gamma^{\prime}\right\}
$$


and thus,

$$
x\left(f^{-1}\left(\gamma^{\prime}\right)\right) \geqq y\left(\gamma^{\prime}\right)
$$

Thus we have shown:

$$
y(\gamma) \leqq x\left(f^{-1}(\gamma)\right) \leqq y(\gamma) \quad \text { for all } \gamma \in \sigma(y)
$$

Therefore,

$$
y=f(x) \text {. }
$$

We would clearly like to develop a theorem such as this for $x$ with a continuous spectrum. To consider this, a somewhat stronger relation than $x \rightarrow y$ will be defined. We will show that the stronger relation, which we will write as $x \stackrel{s}{\longrightarrow} y$, is fully equivalent to the equation $y=f(x)$, with $f$ a continuous function in the induced topology on $R$ on the spectrum of $x$ for an arbitrary observable $x$.

$$
\text { “ } x \stackrel{s}{\longrightarrow} y \text { is Equivalent to } y=f(x) "
$$

We will begin this section with the definition of $x \stackrel{s}{\longrightarrow} y$.

Definition. Assume that $x$ and $y$ are observables. We say $x \stackrel{s}{\longrightarrow} y$ iff there exists an $x$-null set, $N$ (possibly empty), such that for every $\varepsilon>0$ and $\lambda \in \sigma(x) / N$ there exists a $\delta_{\lambda}>0$ such that

$$
\alpha(x)\left(\lambda-\delta_{\lambda}, \lambda+\delta_{\lambda}\right) \leqq \alpha(y)\left(\mu_{\alpha}(y)-\varepsilon, \mu_{\alpha}(y)+\varepsilon\right)
$$

for every state $\alpha$ such that $\operatorname{Var}_{\alpha}(x)<\delta_{\lambda}$.

Intuitively $x \stackrel{s}{\longrightarrow} y$ means that for an arbitrary error $\varepsilon>0$ there is a tolerance $\delta_{\lambda}>0$ such that the probability that $y$ is in the interval

$$
\left(\mu_{\alpha}(y)-\varepsilon, \mu_{\alpha}(y)+\varepsilon\right)
$$

dominates the probability that $x$ is in the interval

$$
\left(\lambda-\delta_{\lambda}, \lambda+\delta_{\lambda}\right)
$$

for any state $\alpha$ in which $x$ can be measured with accuracy $\delta_{\lambda}$. That is, if we measure $x$ accurately, we can measure $y$ accurately. This will be shown in Proposition 6.

Proposition 6. If $x$ and $y$ are observables and if $\|y\|$ exists, then $x \stackrel{s}{\longrightarrow} y$ implies $x \rightarrow y$.

Proof. Let $\varepsilon>0$ and $\lambda \in \sigma(x) / N$ where $N$ is the $x$-null set in 
the definition of $x \stackrel{s}{\longrightarrow} y$. We show that $x \longrightarrow y$ on $\sigma(x) / N$. Now there exists $\delta_{2}>0$ such that

$$
\alpha(x)\left(\lambda-\delta_{\lambda}, \lambda+\delta_{\lambda}\right) \leqq \alpha(y)\left(\mu_{\alpha}(y)-\varepsilon, \mu_{\alpha}(y)+\varepsilon\right)
$$

for every state $\alpha$ for which $\operatorname{Var}_{\alpha}(x)<\delta_{\lambda}$.

For any state $\alpha$ such that $\operatorname{Var}_{\alpha}(x)<\delta_{\lambda}$, we have

$$
\begin{aligned}
\operatorname{Var}_{\alpha}(y)= & \int\left[\nu-\mu_{\alpha}(y)\right]^{2} d \alpha(y)(\nu) \\
= & \int_{\left[\mu_{\alpha}(y)-\sqrt{\varepsilon}, \mu_{\alpha}(y)+\sqrt{\varepsilon]}\right.}\left(\nu-\mu_{\alpha}(y)\right)^{2} d \alpha(y)(\nu) \\
& +\int_{\left\{\left|\mu_{\alpha}(y)-\nu\right|>\sqrt{\varepsilon\}}\right.}\left(\nu-\mu_{\alpha}(y)\right)^{2} d \alpha(y)(\nu) \\
\leqq & \varepsilon \alpha(y)\left(\mu_{\alpha}(y)-\sqrt{\varepsilon}, \mu_{\alpha}(y)+\sqrt{\varepsilon}\right) \\
& +4\|y\|^{2} \alpha(y)\left(\left\{\nu|| \nu-\mu_{\alpha}(y) \mid>\sqrt{\varepsilon}\right\}\right) \\
\leqq & +4\|y\|^{2} \alpha(x)\left(\left\{\nu|\nu-\lambda|>\delta_{\alpha}\right\}\right) .
\end{aligned}
$$

On the other hand, Chebyshev's inequality implies that

$$
\delta_{\lambda} \alpha(x)\left(\left\{\nu|| \nu-\lambda \mid>\delta_{\lambda}\right\}\right) \leqq \operatorname{Var}_{\alpha}(x)
$$

and thus that

$$
\operatorname{Var}_{\alpha}(y) \leqq \varepsilon+4\|y\|^{2} /\left(\delta_{\hat{\lambda}}^{2}\right) \operatorname{Var}_{\alpha}(x) .
$$

Now if we let $\delta_{\lambda}^{\prime}=\min \left\{\delta_{\lambda}, \delta_{\lambda}^{2} \varepsilon /\left(4\|y\|^{2}\right)\right\}$, then $\delta_{\lambda}^{\prime}>0$ and for any state $\alpha$ for which $\operatorname{Var}_{\alpha}(x)<\delta_{\lambda}^{\prime}$ we see that

$$
\operatorname{Var}_{\alpha}(y) \leqq \varepsilon+4\|y\|^{2} \delta_{\lambda}^{\prime} / \delta_{\lambda}^{2} \leqq 2 \varepsilon .
$$

It follows that $x \rightarrow y$.

Proposition 7. Let $x$ be an observable and $f: \sigma(x) \rightarrow \boldsymbol{R}$ a bounded function which is continuous on the complement of an $x$-null set. Then

$$
x \stackrel{s}{\longrightarrow} f(x)
$$

Proof. Let $N$ denote the set of discontinuties of $f$ and $M=$ $\sup _{t \in o(x) / N}|f(t)|$. For each $\varepsilon>0$ and $\lambda \in \sigma(x) / N$ there exists $\delta_{\lambda}>0$ such that $|t-\lambda|<\delta_{\lambda}$ implies

$$
|f(t)-f(\lambda)|<\varepsilon / 3 .
$$

It $\alpha$ is any state, we have by Chebyshev's inequality 


$$
\begin{aligned}
\left|\mu_{\alpha}(f(x))-f(\lambda)\right| & =\left|\int f(t) d \alpha(x)(t)-\int f(\lambda) d \alpha(x)(t)\right| \\
& \leqq \int|f(t)-f(\lambda)| d \alpha(x)(t) \\
& \leqq \varepsilon / 3+2 M \alpha(x)\left(\left\{t|| \lambda-t \mid>\delta_{\lambda}\right\}\right) \\
& \leqq \varepsilon / 3+2 M \operatorname{Var}_{\alpha}(x) /\left(\delta_{\lambda}^{2}\right) .
\end{aligned}
$$

Now let $\delta_{\lambda}^{\prime}=\min \left\{\delta_{\lambda}, \delta_{\lambda}^{2} \varepsilon /(6 M)\right\}$. If $\alpha$ is a state such that $\operatorname{Var}_{\alpha}(x)<$ $\delta_{\lambda}^{\prime}$, then

$$
2 M /\left(\delta_{\lambda}^{2}\right) \operatorname{Var}_{\alpha}(x)<\varepsilon / 3
$$

and $\left|\mu_{\alpha}(f(x))-f(\lambda)\right|<2 / 3 \varepsilon$.

Let $C=\sigma(x) / N$ and $y=f(x)$, then

$$
\begin{aligned}
\left(\lambda-\delta_{\lambda}^{\prime}, \lambda+\delta_{\lambda}^{\prime}\right) \cap C & \subset f^{-1}(f(\lambda)-\varepsilon / 3, f(\lambda)+\varepsilon / 3) \\
& \subset f^{-1}\left(\mu_{\alpha}(y)-\varepsilon, \mu_{\alpha}(y)+\varepsilon\right)
\end{aligned}
$$

and

$$
\begin{aligned}
x\left(\lambda-\delta_{\lambda}^{\prime}, \lambda+\delta_{\lambda}^{\prime}\right) & =x\left(\left(\lambda-\delta_{\lambda}^{\prime}, \lambda+\delta_{\lambda}^{\prime}\right) \cap C\right) \\
& \leqq x\left(f^{-1}\left(\mu_{\alpha}(y)-\varepsilon, \mu_{\alpha}(y)+\varepsilon\right)\right) \\
& =f(x)\left(\mu_{\alpha}(y)-\varepsilon, \mu_{\alpha}(y)+\varepsilon\right)
\end{aligned}
$$

Thus,

$$
\alpha(x)\left(\lambda-\delta_{\lambda}^{\prime}, \lambda+\delta_{\lambda}^{\prime}\right) \leqq \alpha(y)\left(\mu_{\alpha}(y)-\varepsilon, \mu_{\alpha}(y)+\varepsilon\right)
$$

hence

$$
x \longrightarrow y
$$

Proposition 8. Let $x$ and $y$ be observables such that $x \rightarrow y$ and let

$$
f: \sigma(x) \longrightarrow \sigma(y)
$$

be the map defined by Proposition 4. If $\varepsilon>0$ and $\lambda \in \sigma(x) / N$, then there exists $\delta_{\lambda}>0$ such that

$$
x\left(\lambda-\delta_{\lambda}, \lambda+\delta_{\lambda}\right) \leqq y(f(\lambda)-\varepsilon, f(\lambda)+\varepsilon) .
$$

Proof. Observe fist that the map

$$
f: \sigma(x) \longrightarrow \sigma(y)
$$

exists. Since $x \stackrel{s}{\longrightarrow} y$ implies $x \longrightarrow y$, we have by Proposition 4 the existence of $f$. Also we know that $f$ is continuous on $\sigma(x) / N$. Let $\varepsilon>0$ be given. Say that $\lambda \in \sigma(x)$. By the definition of $f$ there 
exists $\delta_{\lambda}^{\prime}>0$ such that if $\alpha$ is a state such that

$$
\left|\mu_{\alpha}(x)-\lambda\right|<\delta_{\lambda}^{\prime}
$$

and

$$
\operatorname{Var}_{\alpha}(x)<\delta_{\lambda}^{\prime}
$$

then

$$
\left|\mu_{\alpha}(y)-f(\lambda)\right|<\varepsilon / 2 \text {. }
$$

Moreover, since $x \stackrel{s}{\longrightarrow} y$, there exists $\delta_{\lambda}^{\prime \prime}>0$ such that

$$
\alpha(x)\left(\lambda-\delta_{\lambda}^{\prime \prime}, \lambda+\delta_{\lambda}^{\prime \prime}\right) \leqq \alpha(y)\left(\mu_{\alpha}(y)-\varepsilon / 2, \mu_{\alpha}(y)+\varepsilon / 2\right)
$$

for every state $\alpha$ for which $\operatorname{Var}_{\alpha}(x)<\delta_{\lambda}^{\prime \prime}$. Let

$$
\delta_{\lambda}=1 / 4 \min \left[\delta_{\lambda}^{\prime \prime}, \delta_{\lambda}^{\prime}, 1\right] \text {. }
$$

We intend to show that

$$
x\left(\lambda-\delta_{\lambda}, \lambda+\delta_{\lambda}\right) \leqq y(f(\lambda)-\varepsilon, f(\lambda)+\varepsilon)
$$

by showing that for every state $\alpha$ for which

$$
\alpha(x)\left(\lambda-\delta_{\lambda}, \lambda+\delta_{\lambda}\right)=1
$$

if follows that

$$
\alpha(y)(f(\lambda)-\varepsilon, f(\lambda)+\varepsilon)=1 .
$$

The desired conclusion will then follow.

This is easy; if $\alpha$ is a state such that

$$
\alpha(x)\left(\lambda-\delta_{\lambda, \lambda}+\delta_{\lambda}\right)=1
$$

then we see that

$$
\operatorname{Var}_{\alpha}(x)<4 \delta_{\lambda} \text { and }\left|\mu_{\alpha}(x)-\lambda\right|<\delta_{\lambda}
$$

and

$$
\begin{aligned}
1=\alpha(x)\left(\lambda-\delta_{\lambda}, \lambda+\delta_{\lambda}\right) & \leqq \alpha(y)\left(\mu_{\alpha}(y)-\varepsilon / 2, \mu_{\alpha}(y)+\varepsilon / 2\right) \\
& \leqq \alpha(y)(f(\lambda)-\varepsilon, f(\lambda)+\varepsilon) .
\end{aligned}
$$

Thus the proposition follows.

Proposition 9. Let $x$ and $y$ be observables such that $x \stackrel{s}{\longrightarrow} y$. Then there is a function

$$
f: \sigma(x) \longrightarrow \sigma(y)
$$


which is continuous on the complement of some $x$-null set and which has the property that $y=f(x)$.

Proof. Let

$$
f: \sigma(x) \longrightarrow \sigma(y)
$$

be the function defined by Proposition 4 .

We first show that

$$
x\left(f^{-1}(E)\right) \leqq y(E)
$$

for any open set $E$. For $\gamma \in E$ choose $\varepsilon_{\gamma}>0$ such that

$$
\left(\gamma-\varepsilon_{\gamma}, \gamma+\varepsilon_{\gamma}\right) \subseteq E .
$$

If $C$ denotes the set of points of $\sigma(x)$ at which $f$ is continuous then, for each

$$
\lambda \in f^{-1}\left(\gamma-\varepsilon_{r}, \gamma+\varepsilon_{\gamma}\right) \cap C
$$

there exists $\delta_{\lambda}>0$ such that

$$
\left(\lambda-\delta_{\lambda}, \lambda+\delta_{\lambda}\right) \cap \sigma(x) \subseteq f^{-1}\left(\gamma-\varepsilon_{\gamma}, \gamma+\varepsilon_{\gamma}\right) .
$$

By use of Proposition 8 we see that we can assure that

$$
x\left(\lambda-\delta_{\lambda}, \lambda+\delta_{\lambda}\right) \leqq y\left(f(\lambda)-d_{\lambda}, f(\lambda)+d_{\lambda}\right)
$$

where

$$
d_{\lambda}=\min \left[\left|f(\lambda)-\left(\gamma-\varepsilon_{\gamma}\right)\right|,\left|f(\lambda)-\left(\gamma+\varepsilon_{\gamma}\right)\right|\right] .
$$

But

$$
\left(f(\lambda)-d_{\lambda}, f(\lambda)+d_{\lambda}\right) \subset\left(\gamma-\varepsilon_{r}, \gamma+\varepsilon_{r}\right)
$$

and thus

$$
\begin{aligned}
x\left(\lambda-\delta_{\lambda}, \lambda+\delta_{\lambda}\right) \leqq y\left(f(\lambda)-d_{\lambda}, f(\lambda)+d_{\lambda}\right) & \leqq y\left(\gamma-\varepsilon_{\gamma}, \gamma+\varepsilon_{\gamma}\right) \\
& \leqq y(E) .
\end{aligned}
$$

Now

$$
\begin{aligned}
& f^{-1}\left(\gamma-\varepsilon_{\gamma}, \gamma+\varepsilon_{\gamma}\right) \cap C \\
& \quad \subseteq \cup\left\{\left(\lambda-\delta_{\lambda}, \lambda+\delta_{\lambda}\right) \mid \lambda \in f^{-1}\left(\gamma-\varepsilon_{\gamma}, \gamma+\varepsilon_{\gamma}\right) \cap C\right\}
\end{aligned}
$$

and thus there exists a sequence $\left\{\lambda_{i}\right\}$ in

$$
f^{-1}\left(\gamma-\varepsilon_{\gamma}, \gamma+\varepsilon_{\gamma}\right) \cap C
$$

such that 


$$
f^{-1}\left(\gamma-\varepsilon_{\gamma}, \gamma+\varepsilon_{\gamma}\right) \cap C \subset \bigcup_{i}\left(\lambda_{i}-\delta_{\lambda_{i}}, \lambda_{i}+\delta_{\lambda_{i}}\right)
$$

(recall that a subspace of a separable space is separable). Thus,

$$
\begin{aligned}
x\left(f^{-1}\left(\gamma-\varepsilon_{\gamma}, \gamma+\varepsilon_{\gamma}\right)\right) & =x\left(f^{-1}\left(\gamma-\varepsilon_{\gamma}, \gamma+\varepsilon_{\gamma}\right) \cap C\right) \\
& \leqq \bigcup_{i} x\left(\lambda_{i}-\delta_{\lambda_{i}}, \lambda_{i}+\delta_{\lambda_{i}}\right) \\
& \leqq y(E) .
\end{aligned}
$$

Now $E$ is seperable and

$$
E=\bigcup_{\gamma \in E}\left(\gamma-\varepsilon_{\gamma}, \gamma+\varepsilon_{\gamma}\right),
$$

thus there exists a sequence $\left\{\gamma_{i}\right\}$ in $E$ such that

$$
E=\bigcup_{i}\left(\gamma_{i}-\varepsilon_{\gamma_{i}}, \gamma_{i}+\varepsilon_{\gamma_{i}}\right) \text {. }
$$

Then

$$
f^{-1}(E)=\bigcup_{i} f^{-1}\left(\gamma_{i}-\varepsilon_{\gamma_{i}}, \gamma_{i}+\varepsilon_{\gamma_{i}}\right)
$$

and

$$
\begin{aligned}
x\left(f^{-1}(E)\right) & =x\left(\bigcup_{i} f^{-1}\left(\gamma_{i}-\varepsilon_{\gamma_{i}}, \gamma_{i}+\varepsilon_{\gamma_{i}}\right)\right) \\
& \leqq \bigcup_{i} x\left(f^{-1}\left(\gamma_{i}-\varepsilon_{\gamma_{i}}, \gamma_{i}+\varepsilon_{\gamma_{i}}\right)\right. \\
& \leqq y(E) .
\end{aligned}
$$

It follows that $x\left(f^{-1}(E)\right) \leqq y(E)$ for every open set $E$. Now let $a \in E$. We will show that

$$
x\left(f^{-1}(a)\right) \leqq y(\{a\})
$$

If

$$
\lambda \in f^{-1}(a) \cap C
$$

then by Proposition 8 there exists $\delta_{2}>0$ such that

$$
x\left(\lambda-\delta_{\lambda}, \lambda+\lambda_{\lambda}\right) \leqq y((a-\varepsilon, a+\varepsilon)) .
$$

Since $f^{-1}(a) \cap C$ is separable, there is a sequence $\left\{\lambda_{i}\right\}_{i=1}^{\infty}$ in $f^{-1}(a) \cap C$ such that

$$
f^{-1}(a) \cap C \subset \bigcup_{i}\left(\lambda_{i}-\delta_{\lambda_{i}}, \lambda_{i}+\delta_{\lambda_{i}}\right)
$$

Thus,

$$
\begin{gathered}
x\left(f^{-1}(a)\right)=x\left(f^{-1}(a)+C\right) \leqq \bigcup_{i} x\left(\lambda_{i}-\delta_{\lambda_{i}}, \lambda_{i}+\delta_{\lambda_{i}}\right) \\
\leqq y(a-\varepsilon, a+\varepsilon)
\end{gathered}
$$


Now

$$
x\left(f^{-1}(a)\right) \leqq y(a-\varepsilon, a+\varepsilon)
$$

for arbitrary $\varepsilon$ thus

$$
x\left(f^{-1}(a)\right) \leqq \bigcap_{n=1}^{\infty} y(a-1 / n, a+1 / n)=y(\{a\}) .
$$

Finally, let $E=(a, b)$ be any open interval. Thus

$$
E^{c}=(-\infty, a) \cup\{a\} \cup\{b\} \cup(b,+\infty)
$$

and if

$$
U=(-\infty, a) \cup(b,+\infty)
$$

then $U$ is open and

$$
x\left(f^{-1}(U)\right) \leqq y(U)
$$

and further

$$
\begin{aligned}
& x\left(f^{-1}(a)\right) \leqq y(a) \\
& x\left(f^{-1}(b)\right) \leqq y(b) .
\end{aligned}
$$

If follows that

$$
\begin{aligned}
x\left(f^{-1}\left(E^{c}\right)\right) & =x\left(f^{-1}(U) \cup f^{-1}(a) \cup f^{-1}(b)\right) \\
& =x\left(f^{-1}(U)\right) \cup x\left(f^{-1}(a)\right) \cup x\left(f^{-1}(b)\right) \\
& \leqq y(U) \cup y(\{a\}) \cup y(\{b\}) \\
& =y\left(E^{c}\right) .
\end{aligned}
$$

Thus

$$
y\left(E^{c}\right)^{\prime} \leqq x\left(f^{-1}\left(E^{c}\right)\right)^{\prime}
$$

and

$$
y(E) \leqq x\left(f^{-1}(E)\right)
$$

for every open interval $E$. Clearly

$$
y(E)=x\left(f^{-1}(E)\right)
$$

for any open interval (finite or not) by a minor modification of the above argument. Since every open set is a disjoint union of open intervals

$$
y(E)=x\left(f^{-1}(E)\right)
$$

for every open set $E$. 
To complete the proof of the proposition, observe that the collection

$$
A=\left\{B \in B(\boldsymbol{R}) \mid y(B)=x\left(f^{-1}(B)\right)\right\}
$$

is easily shown to be a $\sigma$ algebra which contains all open sets. Since $B(R)$ is the smallest such $\sigma$ algebra we have that $A=B(R)$ and thus that

$$
y(B)=x\left(f^{-1}(B)\right)
$$

for every Borel set $B$.

We now present the following theorem as a synopsis.

Theorem. Let $x$ and $y$ be observables. Let

$$
f: \sigma(x) \longrightarrow \sigma(y)
$$

be the naturaly induced mapping when $x \rightarrow y$.

(1) $f$ is continuous on the complement of an $x$-null set,

(2) the relation $x \stackrel{s}{\longrightarrow} y$ is equivalent to the functional calculus equation $y=f(x)$,

(3) the relation $x \stackrel{s}{\longrightarrow} y$ implies $x \rightarrow y$, and

(4) if $x$ has a countable spectrum and $x \rightarrow y$ on the complement of $N=\{\lambda \mid x(\lambda)=0\}$, then $y=f(x)$, i.e., $x \rightarrow y$ is equivalent to $x \stackrel{s}{\longrightarrow} y$.

Finally we note that we do not know, in general, whether or not $x \rightarrow y$ implies $y=f(x)$, but we rather suspect that this is not so.

\section{REFERENCES}

George W. Mackey, Mathematical Foundations of Quantum Mechanics, Benjamin Pulb., 1963. New York, New York.

V. S. Varadarajan, Probability in physics and a theorem on simultaneous observability. Comm. of P. Appl. Math. Vol. XV, 1962, 189-217.

Received June 11, 1974 and in revised form October 25, 1974. The work was done in part while I was supported by the NIH (Grant Number ST 1 GM 678) through Dr. Lucas' Biomathematics Programm at NCSU. I am indebted to the NIH and the Biomath, Group. Prof. Ronald Fulp, my advisor, was generous with his time. He always showed interest and encouragement, and his friendship never failed.

North Carolina State University at Raleigh

Current Address: Institut für med. Statistik und Dokumentation der med. Hochschule Lübeck

Direktor: Prof. Dr. med. H. Fassl

24 Lübeck

Ratzeburger Allee 160

West Germany 



\section{PACIFIC JOURNAL OF MATHEMATICS}

\section{EDITORS}

RICHARD ARENS (Managing Editor) University of California

Los Angeles, California 90024

\section{R. A. Beaumont}

University of Washington Seattle, Washington 98105
J. DugundJI Department of Mathematics University of Southern Californıa Los Angeles, California 90007

D. Gilbarg aNd J. Milgram Stanford University Stanford, California 94305

\section{ASSOCIATE EDITORS}

E. F. BeCKENBACH

B. H. NeUmanN

F. Wolf

K. YoSHIDA

\section{SUPPORTING INSTITUTIONS}

UNIVERSITY OF BRITISH COLUMBIA CALIFORNIA INSTITUTE OF TECHNOLOGY UNIVERSITY OF CALIFORNIA MONTANA STATE UNIVERSITY UNIVERSITY OF NEVADA NEW MEXICO STATE UNIVERSITY OREGON STATE UNIVERSITY UNIVERSITY OF OREGON OSAKA UNIVERSITY
UNIVERSITY OF SOUTHERN CALIFORNIA STANFORD UNIVERSITY UNIVERSITY OF TOKYO UNIVERSITY OF UTAH WASHINGTON STATE UNIVERSITY UNIVERSITY OF WASHINGTON AMERICAN MATHEMATICAL SOCIETY NAVAL WEAPONS CENTER 


\section{Pacific Journal of Mathematics \\ Vol. 59, No. 1 \\ May, 1975}

Shashi Prabha Arya and M. K. Singal, More sum theorems for topological

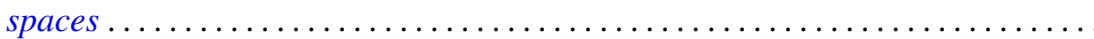

Goro Azumaya, F. Mbuntum and Kalathoor Varadarajan, On M-projective and

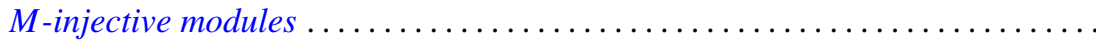

Kong Ming Chong, Spectral inequalities involving the infima and suprema of

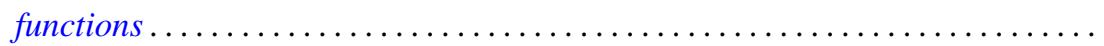

Alan Hetherington Durfee, The characteristic polynomial of the monodromy .......

Emilio Gagliardo and Clifford Alfons Kottman, Fixed points for orientation

preserving homeomorphisms of the plane which interchange two points ......

Raymond F. Gittings, Finite-to-one open maps of generalized metric spaces .......

Andrew M. W. Glass, W. Charles (Wilbur) Holland Jr. and Stephen H. McCleary,

$a^{*}$-closures of completely distributive lattice-ordered groups .............

Matthew Gould, Endomorphism and automorphism structure of direct squares of

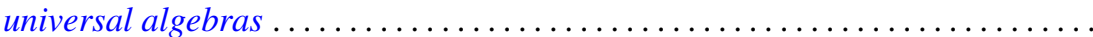

R. E. Harrell and Les Andrew Karlovitz, On tree structures in Banach spaces .....

Julien O. Hennefeld, Finding a maximal subalgebra on which the two Arens

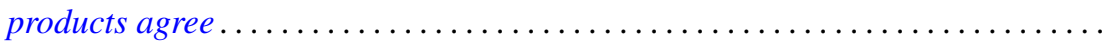

William Francis Keigher, Adjunctions and comonads in differential algebra .......

Robert Bernard Kelman, A Dirichlet-Jordan theorem for dual trigonometric

series

Allan Morton Krall, Stieltjes differential-boundary operators. III. Multivalued operators-linear relations...

Hui-Hsiung Kuo, On Gross differentiation on Banach spaces .

Tom Louton, A theorem on simultaneous observability ...

Kenneth Mandelberg, Amitsur cohomology for certain extensions of rings of algebraic integers.

Coy Lewis May, Automorphisms of compact Klein surfaces with boundary . . .

Peter A. McCoy, Generalized axisymmetric elliptic functions .

211

Muril Lynn Robertson, Concerning Siu's method for solving $y^{\prime}(t)=F(t$, $y(g(t)))$. .

Richard Lewis Roth, On restricting irreducible characters to normal

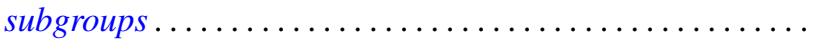

Albert Oscar Shar, $P$-primary decomposition of maps into an $H$-space .

Kenneth Barry Stolarsky, The sum of the distances to certain pointsets on the unit circle.

Bert Alan Taylor, Components of zero sets of analytic functions in $C^{2}$ in the unit ball or polydisc

Michel Valadier, Convex integrands on Souslin locally convex spaces ...

Januario Varela, Fields of automorphisms and derivations of $C$

Arnold Lewis Villone, A class of symmetric differential operators with deficiency

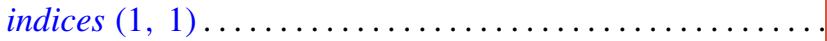

